Journal of Animal and Veterinary Advances 11 (17): 3156-3159, 2012

ISSN: $1680-5593$

(C) Medwell Journals, 2012

\title{
Isolation and the Analysis of 16S rDNA Sequence of Swine Bordetella bronchiseptica
}

\author{
Zuo Zhicai, Fu Tongchao, Wan Hongping, Dai Hongbo, \\ Chen Lei, Xu Zhiwen and Zhu Lin \\ Sichuan Agricultural University, Ya'an, 625014 Sichuan, P.R. China
}

\begin{abstract}
A strain named SCBI-1, isolated from a sick pig was diagnosed by cultural characteristics and morphologic observation with its pathogenicity studied by mice virulent experiment and was accurately identified by biochemical assay and the analysis of $16 \mathrm{~S}$ rDNA sequence. Drug resistance was analysed by sensitive assay. Homology of the 16S rDNA between SCBI-1 and Bordetella bronchiseptica is $99.3-99.9 \%$, the study successfully isolated a strain of Bordetella bronchiseptica which could lead a mouse to death within $16 \mathrm{~h}$ and is resistant to penicillin, rifampicin, clindamycin, ampicillin and cefalotin.
\end{abstract}

Key words: Swine Bordetella bronchiseptica, biochemical assay, $16 \mathrm{~S}$ rDNA, sensitive assay, penicillin, mouse

\section{INTRODUCTION}

Bordetella bronchiseptica, the Gram-negative bacterial pathogen and the etiologic agent of upper respiratory infections in a wide range of mammalian hosts, is associated with a number of veterinary syndromes such as atrophic rhinitis and pneumonia in pigs (Burgos et al., 2010; Sukumar et al., 2010; Nicholson et al., 2009). Atrophic Rhinitis (AR) is divided into non-progressive $\mathrm{AR}$ which is caused by $B$. bronchiseptica and progressive $\mathrm{AR}$ which is caused by toxigenic strains of Pastrurella multocida (Giles, 1986). B. bronchiseptica could produce the dermonecrotic toxin which induces mucosal damage in swine nasal tissue and causes turbinate atrophy and pneumonic lesions characterized by necrosis, hemorrhage, neutrophil accumulation and eventually brosis (Brockmeier and Register, 2007). Meanwhile, it could infect humans, especially people who have weak immune system such as AIDS patient which highly attracts international attention (Valencia, 2004).

A disease characterized by cough, difficult breath, necrosis, hemorrhage and pneumonia occurred in a pig farm in Mingshan of Sichuan province. The lung and liver tissue samples of a swine herd which had the clinical signs and gross pathological lesions were collected sterilely for pathogenic detection.

The current research was to isolate a suspicious strain of B. bronchiseptica from a representative sick pig featured by the clinical signs and gross pathological lesions and identify the isolated strain based on morphology, chemical characteristics and genomic sequence analysis.

\section{MATERIALS AND METHODS}

Bacterial isolate and culture conditions: The lung and liver tissue samples of a swine herd which had clinical signs and gross pathological lesions were collected sterilely for pathogenic detection. The samples were firstly grown on Tryptose Soya Agar (TSA) plates supplemented with $5 \%$ fetal calf serum (Gibco) and $0.01 \%$ Nicotinamide Adenine Dinucleotide (NAD). Plates were incubated at $37^{\circ} \mathrm{C}$ in $5 \% \mathrm{CO}_{2}$ for $24-48 \mathrm{~h}$. Colony, characterized by smooth, moist, semi-transparent and dew size were extracted to purification cultivation. The isolated and purified suspicious strain, named after SCBI-1. The pure culture was kept for futher study.

Cultural characteristics assay: The isolates were respectively cultivated on Luria-Bertani plates, MacConkey agar plates and blood agar plates at $37^{\circ} \mathrm{C}$ in $5 \% \mathrm{CO}_{2}$ for $24-48 \mathrm{~h}$. Growth situation on different plates were observed and recorded.

Morphologic observation: To observe the morphology of the isolates, a solo purified strain was extracted to Gram stain.

Virulence test in mice: The project was supervised and supported by China Animal Protection Association. Twelve mice (each weighted about $20 \mathrm{~g}$ ) were randomly divided into three groups named after A-C. The three groups were respectively injected with $0.2 \mathrm{~mL}$ bacterium suspension using PBS to dilute to $10^{9} \mathrm{CFU} \mathrm{mL}^{-1}, 0.2 \mathrm{~mL}$ Luria-Bertani broth without any bacterium and $0.2 \mathrm{~mL}$ PBS. The three groups were raised separately with careful

Corresponding Author: Zuo Zhicai, Sichuan Agricultural University, Ya'an, 625014 Sichuan, P.R. China 
observation. The dead mice were instantly dissected, pathological variation was recorded. The liver and lung tissue of the dead mice were sampled sterilely to identify the pathogens.

Biochemical assay: Glucose, mannose, sucrose, lactose, arabinose, maltose, sorbitol, MR, indole, oxidase, catalase and citric acid were used to test the biochemical characteristics of SCBI-1.

PCR amplification of $16 S r D N A$ gene: Total genomic DNA was extracted by boiling method and supernatant was used for PCR amplification. The 16S rDNA gene expected to $1500 \mathrm{bp}$ was amplified using the universal primers, forward: 5'-AGAGTTTGATCCTGGCTCAG-3' and reverse: 5'-GGTTACCTTGTTACGACTT-3' (Bal and Bal, 2012). PCR was performed in a $20 \mu \mathrm{L}$ reaction mixture containing: $1.0 \mu \mathrm{L}$ of DNA template, $10.0 \mu \mathrm{L}$ of $2 \times \mathrm{Taq} \mathrm{PCR}$ Master Mix, $8.0 \mu \mathrm{L}$ of $\mathrm{ddH}_{2} \mathrm{O}, 0.5 \mu \mathrm{L}$ of each primer. Amplification conditions were as follows: an initial denaturation step at $95^{\circ} \mathrm{C}$ for $5 \mathrm{~min}$ followed by 30 cycles of denaturation at $95^{\circ} \mathrm{C}$ for $35 \mathrm{sec}$ annealing at $55^{\circ} \mathrm{C}$ for $40 \mathrm{sec}$, polymerization at $72^{\circ} \mathrm{C}$ for $1.5 \mathrm{~min}$ and a final extension at $72^{\circ} \mathrm{C}$ for $7 \mathrm{~min}$. Amplified bands were separated by gel electrophoresis ( $1 \%$ agarose gel) and visualized on a gel documentation system. According to the manufacturer's instructions, the QIAquick Gel Extraction kit was used to collect PCR products which were subsequently connected with pMD19-T simple Vector. The ligation reaction mixture were transferred into DH5 $\alpha$ competent cells.

DNA sequencing and homology analysis: Plasmid Extraction kit was used to extract recombinant plasmid. The positive recombinant plasmid were sent to Takara for sequencing. Sequences were compared to the sequences available in the GenBank database using BLAST. Then, homology of sequences was analysed and a phylogenetic tree was constructed by DNAStar Software.

Drug sensitive assay: Ceftriaxone, cefotaxime, cefuroxime, norfloxacin, SMZco, cefazolin, cefoperazone, cephaloridine IV, vancomycin, gentamycin were employed to test the sensitivity of the purified strains about medicines judging by diameter inhibition zone.

\section{RESULTS AND DISCUSSION}

Cultural characteristics, morphology and virulence: SCBI-1 could grow on Luria-Bertani plates, MacConkey agar plates and blood agar plates. Meanwhile, SCBI-1 is a Gram-negative, characterized by single, consistent
Table 1: The results of biochemical assay

\begin{tabular}{lclclc}
\hline Reagents & Results & Reagents & Results & Reagents & Results \\
\hline Glucose & - & Mannose & - & Sucrose & - \\
Lactose & - & Arabinose & - & Maltose & - \\
Sorbitol & - & MR & - & Indole & - \\
Oxidase & + & Catalase & + & Citric acid & + \\
\hline
\end{tabular}

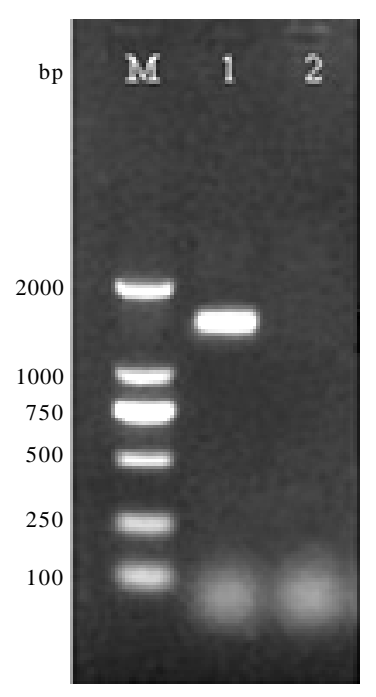

Fig. 1: Electrophoresis figure of PCR product. M: Molar mass marker; 1: PCR product; 2: Negative contrast

morphology without capsule, flagella and spore nearly roundness and dyeing both of ends. Furthermore, mice challenged with SCBI-1 were dead within $16 \mathrm{~h}$ however, the other two groups were normal. The bacterium corresponding to isolates were isolated from the dead mice which indicated that SCBI-1 is virulent.

Biochemical assay: SCBI-1 did not ferment and decompose any carbohydrates. MR and indole test were negative. Oxidase, catalase and citric acid test were positive (Table 1). It accorded with B. bronchiseptica in Bergey's manual of systematic bacteriology (Dong and Cai, 2001).

PCR amplification of $16 S r D N A$ gene: The PCR was successfully amplified an amplicon as predicted size 1500 bp (Fig. 1).

16S rDNA gene sequencing and homology analysis: According to the BLAST, the homology of the $16 \mathrm{~S}$ rDNA sequence between SCBI-1 and Bordetella is high. Further comparision between SCBI-1 and two Bordetella and several gram negative bacterium via DNAStar Software indicates that the identity of the $16 \mathrm{~S}$ rDNA sequence between SCBI-1 and Bordetella bronchiseptica is highly $99.9 \%$ while others is lower (Table 2). 
J. Anim. Vet. Adv., 11 (17): 3156-3159, 2012

Table 2: The homologous comparision of the $16 S \mathrm{~S}$ DNA gene among SCBI-1 and two Bordetella and several gram negative bacterium

\begin{tabular}{|c|c|c|c|c|c|c|c|c|c|c|c|c|c|c|c|}
\hline Species & 1 & 2 & 3 & 4 & 5 & 6 & 7 & 8 & 9 & 10 & 11 & 12 & 13 & 14 & 15 \\
\hline 1 & - & 99.4 & 74.4 & 73.9 & 74.4 & 77.5 & 75.9 & 72.9 & 76.4 & 77.5 & 72.6 & 72.9 & 74.7 & 73.9 & 99.5 \\
\hline 2 & 0.0 & - & 72.8 & 72.3 & 73.3 & 76.4 & 75.2 & 72.3 & 76.6 & 76.4 & 71.6 & 71.6 & 73.8 & 73.6 & 99.3 \\
\hline 3 & 21.2 & 21.5 & - & 99.5 & 85.6 & 85.8 & 92.0 & 92.7 & 84.9 & 85.1 & 93.0 & 93.0 & 84.9 & 83.6 & 72.2 \\
\hline 4 & 21.3 & 21.6 & 0.1 & - & 85.2 & 85.4 & 91.5 & 92.2 & 84.5 & 84.7 & 92.5 & 92.5 & 84.5 & 83.1 & 68.4 \\
\hline 5 & 20.7 & 21.3 & 12.9 & 12.9 & - & 98.6 & 85.6 & 84.6 & 94.2 & 95.3 & 84.9 & 85.2 & 97.1 & 97.0 & 75.7 \\
\hline 6 & 20.3 & 20.8 & 12.6 & 12.6 & 0.7 & - & 86.0 & 85.4 & 93.6 & 95.7 & 85.6 & 85.9 & 96.3 & 96.4 & 71.5 \\
\hline 7 & 20.6 & 20.9 & 6.5 & 6.6 & 13.0 & 12.5 & - & 98.8 & 85.0 & 85.1 & 93.6 & 93.6 & 85.6 & 86.0 & 74.0 \\
\hline 8 & 20.8 & 21.0 & 6.5 & 6.7 & 12.9 & 12.4 & 0.5 & - & 84.9 & 85.0 & 94.3 & 94.4 & 84.6 & 85.0 & 73.8 \\
\hline 9 & 21.7 & 21.0 & 13.1 & 13.3 & 4.6 & 5.3 & 13.5 & 13.4 & - & 97.9 & 84.7 & 83.3 & 95.5 & 95.3 & 77.3 \\
\hline 10 & 20.6 & 21.1 & 13.1 & 13.3 & 3.5 & 4.0 & 13.5 & 13.3 & 1.4 & - & 85.2 & 85.6 & 95.5 & 95.3 & 77.1 \\
\hline 11 & 21.5 & 22.1 & 6.7 & 6.9 & 13.0 & 12.8 & 5.2 & 5.1 & 13.3 & 12.9 & - & 99.9 & 85.1 & 84.4 & 72.7 \\
\hline 12 & 21.2 & 21.9 & 6.7 & 6.9 & 12.7 & 12.4 & 5.1 & 5.0 & 14.5 & 12.7 & 0.1 & - & 85.1 & 84.4 & 72.5 \\
\hline 13 & 20.3 & 20.9 & 13.4 & 13.6 & 2.5 & 3.1 & 13.1 & 13.1 & 3.7 & 3.7 & 13.1 & 13.1 & - & 99.4 & 77.2 \\
\hline 14 & 20.6 & 20.7 & 13.7 & 13.9 & 2.4 & 3.1 & 13.2 & 13.2 & 3.9 & 3.9 & 13.8 & 13.8 & 0.4 & - & 77.4 \\
\hline 15 & 0.1 & 0.1 & 24.9 & 25.1 & 21.5 & 21.3 & 23.1 & 23.6 & 21.2 & 21.5 & 24.7 & 24.5 & 20.6 & 20.6 & - \\
\hline
\end{tabular}

1: B. bronchiseptica, X57026; 2: Bordetella bronchiseptica, NR025949; 3: Acbnobacillus pleuropneumonia, AF 03305; 4: Acbnobacillus pleuropneumonia, NR044752; 5: E. coli EHEC Strains ATCC 43895 Z83205; 6: E. coli Strains KCTC 2441. EU014689; 7: H. parasuis, FJ667962; 8: H. parasuis, FJ667982; 9: Klebsiella, DQ 831003; 10: Klebsiella, EU545402; 11: Pasteurella, FJ405340; 12: Pasteurella muttocida, AF224297; 13: Salmonella enterica, AF227869; 14: Salmonella enterica, AF332600; 15: SCBI-1

Table 3: The result of the sensitive test

\begin{tabular}{lcl}
\hline Drug name & Diameter of inhibition $(\mathrm{mm})$ & Results \\
\hline Amikacin & 22 & Susceptible \\
Ciprofloxacin & 23 & Susceptible \\
Penicillin & - & Resistance \\
Kananycin & 10 & Moderately sensitive \\
Rifampicin & - & Resistance \\
Clindamycin & - & Resistance \\
Gentamycin & 17 & Moderately sensitive \\
Levofloxacin & 17 & Moderately sensitive \\
Ampicillin & - & Resistance \\
Cefalotin & - & Resistance \\
\hline
\end{tabular}

-: Means no inhibition zone

A phylogenetic tree was constructed based on Clustalx program, revealing that SCBI-1 and Bordetella bronchiseptica were on the same branch (Fig. 2). It deduces that SCBI-1 belongs to Bordetella bronchiseptica.

Sensitive assay: Sensitive assay showed that SCBI-1, Bordetella bronchiseptica is susceptible to amikacin, ciprofloxacin; moderately sensitive to kanamycin, gentamycin, levofloxacin and resistance to penicillin, rifampicin, clindamycin, ampicillin and cefalotin (Table 3 ).

The study faced great obstacles because of the small amount of wanted bacterium that grow slowly in pathological tissues and the interference of other microbe which reduced separation rate. However, the research isolated a suspicious strain, named after SCBI-1 from the typical parts with gross pathological lesions. The Gram-negative SCBI-1 could grow on Luria-Bertani plates, MacConkey agar plates and blood agar plates. Gram stain shows that the isolated strain is single, consistent morphology without capsule, flagella and spore nearly roundness and dyeing both of ends. Yet there are some defects that the result of gram stain may be confused with Enterobacteriaceae and it is hard to predict the phylogeny

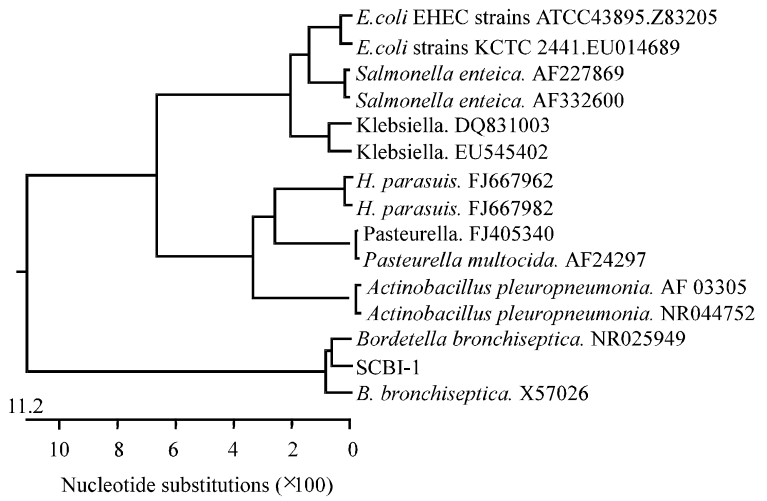

Fig. 2: Dendrogram of genetic relationships among $16 \mathrm{~S}$ rDNA genotypes of bacteria classified. The tree showed the relationship based on partial sequences of the $16 \mathrm{~S} r R N A$ gene of selected clones. The sequence alignment was performed by means of the CLUSTAL X program and the tree was generated by the Neighbor-Joining Method

only based on biochemical assay. The $16 S r D N A$ gene as an operational taxonomic unit (Jinsheng et al., 2011) is selected to identify the suspicious strain in this study. The 16S rDNA gene analysis which provides taxonomic relatedness and an estimate of the evolutionary distance between sequences is widely used to investigate the relationships of prokaryotes (Raje et al., 2010).

Based on cultural characteristics, morphology, mice virulent experiment, biochemistry assay and the analysis of $16 \mathrm{~S} \mathrm{rDNA}$ sequence that the homology of the 16S rDNA between SCBI-1 and Bordetella bronchiseptica is $99.3-99.9 \%$, the study successfully isolated a strain of B. bronchiseptica which is susceptible to amikacin, ciprofloxacin; moderately sensitive to 
kanamycin, gentamycin, levofloxacin and resistance to penicillin, rifampicin, clindamycin, ampicillin, cefalotin. It indicates that $B$. bronchiseptica is highly drug-resistant and the more antibiotics are used the stronger drugresistance is.

\section{CONCLUSION}

Therefore medicines, especially antibiotics should be used scientifically and alternately. Thus, this study offers materials to the exploration of $B$. bronchiseptica diagnostic techniques and vaccines.

\section{REFERENCES}

Bal, E.B.B. and M.A. Bal, 2012. Effects of chemical additives and ensiling time on whole plant wheat silage microbial profiles inferred by phenotypic and $16 \mathrm{~S}$ ribosomal DNA analyses. World J. Microbiol. Biotechnol., 28: 767-776.

Brockmeier, S.L. and K.B. Register, 2007. Expression of the dermonecrotic toxin by Bordetella bronchiseptica is not necessary for predisposing to infection with toxigenic Pasteurella multocida. Vet. Microbiol., 125: 284-289.

Burgos, J.M., N.D. King-Lyons and T.D. Connell, 2010. Expression of $\mathrm{BfrH}$, a putative siderophore receptor of Bordetella bronchiseptica, is regulated by iron, fur1 and the extracellular function sigma factor EcfI. Infec. Immun., 78: 1147-1162.

Dong, X.Z. and M.Y. Cai, 2001. A Systematic Manual of Determinative Bacteriology. Chinese Scientific Press, Beijing, China, pp: 9-348.
Giles, C. J., 1986. Atrophic Rhinitis. In: Diseases of Swine, Leman, A. D., O. R. Glock, B. Straw, W.L. Mengeling, R.H.C. Penny and E. Scholl (Eds.). 6th Edn., Iowa State University Press, Ames, IA, USA., pp: 437-469.

Jinsheng, S., G. Fei, G. Xuyun, W. Junli, L. Xiang and L. Jingjing, 2011. Seasonal changes and diversity of bacteria in Bohai Bay by RFLP analysis of PCR-amplified $16 S$ rDNA gene fragments. World J. Microbiol. Biotechnol., 27: 275-284.

Nicholson, T.L., A.M. Buboltz, E.T. Harvill and S.L. Brockmeier, 2009. Microarray and functional analysis of growth phase-dependent gene regulation in Bordetella bronchiseptica. Infec. Immun., 77: 4221-4231.

Raje, D. V., H. J. Purohit, Y. P. Badhe, S. S. Tambe and B.D. Kulkarni, 2010. Self-organizing maps: A tool to ascertain taxonomic relatedness based on features derived from 16S rDNA sequence. J. Biosci., 35: 617-627.

Sukumar, N., G. P. Sloan, M. S. Conover, C. F. Love, S. Mattoo, N. D. Kock and R. Deora, 2010. Cross-species protection mediated by a Bordetella bronchiseptica strain lacking antigenic homologs present in acellular pertussis vaccines. Infec. Immun., 78: 2008-2016.

Valencia, M.E., A Enriquez, N. Camino and V. Moreno, 2004. Bordetella bronchiseptica pneumonia in patients with HIV. Enferm. Infect. Microbiol. Clin., 22: $502-503$. 